\title{
Coaxial Hyperplanes
}

\author{
T. Srinivasarao \\ Asst. Professor, Dept of Math \\ University College of Science \& Technology \\ Adikavi Nannaya University \\ AP, India
}

\author{
K. Revathi \\ Asst. Professor, Dept of Math \\ University College of S \& T \\ Adikavi Nannaya University \\ AP, India
}

\begin{abstract}
For Euclidean geometry, the basis vectors are the source of inspiration for mutually perpendicular/orthogonal concepts that rules over the world in the form of tangent, normal and binormal or the rectangular co-ordinate system. So, the Pythagoras theorem is valid in 3 - dimensions. But when the $4^{\text {th }}$ and higher dimensional space does not obey Pythagoras and thus the higher ideas like 'norm', 'ortho - normal basis' and more are required. So, the plane in a Euclidean geometry becomes a 'Hyperplane' in the $n$ dimensional space for $n>3$. Pivoting in the linear programming or the construction of ortho-normal basis using Gram - Schmidt's process and the $p^{\text {th }}$ norm are some of the methods that scales to the required heights. In the astronomical discussions, the planets in the solar system will come as close as possible and the distance required to go round and reach back is an idea or the thought source in which the shortest distance between two hyperplanes is presented.
\end{abstract}

\section{Hyper Plane:}

\section{INTRODUCTION:}

In an $n$-dimensional linear space, how many hyper - planes would be there, of $n-1$-dimensional those form a family of symmetric subspaces?

Introduction: planes through origin only will be subspaces. But the collection of parallel planes cannot form the collection of subspaces while one and only one plane will have the origin on it. For instance,

$$
a_{1} x_{1}+a_{2} x_{2}+\ldots+a_{n-1} x_{n-1}+a_{n} x_{n}=c
$$

will be a hyperplane in $n$-dimensional space $V_{n}(F)$ for $c$ is any constant and when $c=0$,

the set of vectors $x_{1}, x_{2}, \ldots, x_{n}$ that satisfy the condition (1.1) will form the subspace showing $a_{1} \neq 0, a_{2} \neq 0, a_{n-1} \neq 0, a_{n}=0$ whenever $c \neq 0$. In the case of $c=0$, if $a_{i}=0 \forall 1 \leq i \leq n$, then $\left\{x_{1}, x_{2}, \ldots, x_{n-1}\right\}$ will be the basis of the subspace which is a hyperplane through the origin.

See that $\pi_{j}=\alpha_{1} x_{1}+\alpha_{2} x_{2}+\ldots+\alpha_{n} x_{n}=0, \alpha_{k}, 1 \leq k \leq n, k \neq i$

are the hyper planes that contain the $X_{i}$ - axis as the axis of intersection or common axis or the axis of rotation for different non zero values of $\alpha_{k}$ and $\left(\sum_{j=1}^{n} \alpha_{j}^{n}\right)^{1 / n}=1$

Definition: a family of hyperplanes through a common line in $V_{n}(F)$ is a co-axial family of hyperplanes.

There are infinitely many co-axial families of hyperplanes through origin. A class of co-axial families will have co-ordinate axes as the axes of symmetry and other families will have lines through origin as axes of symmetry.

Definition: a non - homogeneous co-axial family of hyperplanes are the family that have the line of symmetry not through the origin.

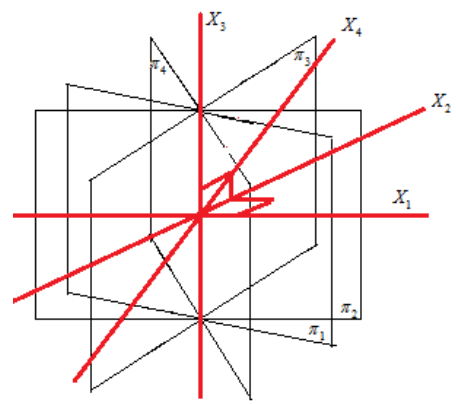


There will be a unique plane through origin in the family of non - homogeneous co-axial family of hyperplanes.

The $X_{i}$ - axis can be represented by $X_{1}=0, X_{2}=0, \ldots, X_{i-1}=0, X_{i+1}=0, \ldots, X_{n}=0$

\section{Line of Intersection of Hyper Planes:}

Two hyper planes $\pi_{r}=\alpha_{1} x_{1}+\alpha_{2} x_{2}+\ldots+\alpha_{n} x_{n}=0$ and

$\pi_{s}=\beta_{1} x_{1}+\beta_{2} x_{2}+\ldots+\beta_{n} x_{n}=0$ are clearly having the common line $X_{i}$ and so are not coincident if and only if

$\frac{\alpha_{k}}{\beta_{k}} \neq \frac{\alpha_{m}}{\beta_{m}}$

for at least one distinct pair $k, m$ among $1,2, \ldots, i-1, i+1, \ldots, n$.

Any line not coincident with any coordinate axis will be of the axis of rotation for the family of the hyper planes

$$
\left\{\pi_{j}=\alpha_{1} x_{1}+\alpha_{2} x_{2}+\ldots+\alpha_{i-1} x_{i-1}+\alpha_{i} x_{i}+\alpha_{i+1} x_{i+1}+\ldots+\alpha_{n} x_{n}=0\right\}
$$

If a line $\lambda$ is not through origin in $V_{n}(F)$, then it can be parametrized as

$$
x_{1}=\alpha_{1} t+\beta_{1}, x_{2}=\alpha_{2} t+\beta_{2}, \ldots, x_{n}=\alpha_{n} t+\beta_{n}
$$

Two lines $\lambda_{1}$ defined by $x_{1}=\alpha_{1} t+\beta_{1}, x_{2}=\alpha_{2} t+\beta_{2}, \ldots, x_{n}=\alpha_{n} t+\beta_{n}$ and $\lambda_{2}$ defined by $x_{1}=\gamma_{1} t+\delta_{1}, x_{2}=\gamma_{2} t+\delta_{2}, \ldots, x_{n}=\gamma_{n} t+\delta_{n}$ that are not intersecting if $\frac{\alpha_{k}}{\gamma_{k}} \neq \frac{b_{k}}{\delta_{k}}$ for at least one $k$ such that $1 \leq k \leq n$.

Definition: If $\lambda_{1}$ and $\lambda_{2}$ are the lines on two different members of a family of hyperplanes that do not intersect, then they are skew hyper lines on hyperplanes.

Theorem: Two skew hyper lines in different co-axial families will be on a unique pair of parallel hyperplanes and so, the distance between the planes will be the shortest distance between the lines.

Proof: there is a unique position in which the line $\lambda_{1}$ is in the plane

$$
\begin{aligned}
& \pi_{1}=\alpha_{1} x_{1}+\alpha_{2} x_{2}+\ldots+\alpha_{i-1} x_{i-1}+\alpha_{i+1} x_{i+1}+\ldots+\alpha_{n} x_{n}=0 ; \text { and the line } \lambda_{2} \text { is in } \\
& \pi_{2}=\beta_{1} x_{1}+\beta_{2} x_{2}+\ldots+\beta_{i-1} x_{i-1}+\beta_{i+1} x_{i+1}+\ldots+\beta_{n} x_{n}=c, c \neq 0 \text { such that } \frac{\alpha_{k}}{\beta_{k}}=\frac{\alpha_{m}}{\beta_{m}} \text { for all } 1 \leq k, m \leq n
\end{aligned}
$$

Since $\lambda_{1}$ and $\lambda_{2}$ are the skew hyper lines, at one instance, these lines will be as close as possible and the next moment, the distance between them will be more and keep increasing between any two points, each is on either the lines.

In other words, there is a unique pair of points $p_{1} \in \lambda_{1}$ and $p_{2} \in \lambda_{2}$ such that $\left\|p_{2}-p_{1}\right\|_{n}$ will be the shortest distance between the given lines.

It can be followed that the line of minimum distance between $\lambda_{1}$ and $\lambda_{2}$ is $\lambda_{3}=\overleftrightarrow{p_{1} p_{2}}$ such that $\lambda_{3}$ is normal to both $\lambda_{1}$ and $\lambda_{2}$. So, the line $\lambda_{3}$ is through the feet of the normal that are the points of intersection of the lines namely $p_{1} \in \lambda_{1}$ and $p_{2} \in \lambda_{1}$ Incidentally $\lambda_{1} \in \pi_{1}$ and $\lambda_{2} \in \pi_{2}$ where $\pi_{1}$ is in one co - axial system and $\pi_{2}$ is in the other co - axial system such that $\pi_{1} \& \pi_{2}$ will be parallel.

The distance between the feet of the normal skew hyper line is the distance between the parallel planes $\pi_{1}$ and $\pi_{2}$.

The minimum distance possible between the planes $\pi_{1} \& \pi_{2}$ the length of the skew hyper line of shortest distance is

$$
\frac{|c|}{\left(\sum_{j=1}^{n}\left(\alpha_{j}-\beta_{j}\right)^{n}\right)^{1 / n}}, 1 \leq i \leq n
$$


Note: if both co - axial systems are not through origin, then the shortest distance between the skew hyper lines $\left\|d_{1}-d_{2}\right\|$

$\left[\sum_{j=1}^{n}\left(\alpha_{j}-\beta_{j}\right)^{n}\right]^{1 / n}$

\section{REFERENCES}

1. Charles w.Curtis(1968) Linear Algebra, page 62, Allyn\&Bacon, Boston

2. Victor V.Prasolov \& VM Tikhomirov(1997, 2001) Geometry, page 22, Vol. 200 in Transactions of Mathematical Monographs, American Mathematical Society

3. Heinrich Guggenheimer(1977) Applicable Geometry, page 7, Krieger, Huntington ISBN 0-88275-368-1

4. Berger, Marcel(1987), Geometry 1, Berlin; Springer, ISBN 3-540 - 11658-3

5. Brannan, David A; Esplen, Matthew F; Gray, Jeremy J(1999), Geometry, Cambridege University Press, ISBN 978-0-521-44177-3 